\title{
Arterial Pandemonium: A Case Report of Pan-Aortic Dissection Masquerading as an Inferior Myocardial Infarction, Stroke and Bilateral Lower Extremity Paraplegia
}

\author{
Injoon Lee ${ }^{1 *}$, Matthew P. Cauchi' ${ }^{1}$ Amitabh Parashar ${ }^{2}$
}

'Virginia Tech-Carilion Clinic School of Medicine, Department of General Cardiovascular Disease, Roanoke, VA, USA

${ }^{2}$ Salem Veterans Affairs Medical Center, Department of Cardiology, Salem VA, USA

\section{Article Info}

\section{Article Notes}

Received: December 31, 2019

Accepted: January 31, 2020

\section{*Correspondence:}

*Dr. Injoon Lee, Virginia Tech-Carilion Clinic School of Medicine, Department of General Cardiovascular Disease, Roanoke, VA, USA; Email: ilee@carilionclinic.org.

C 2020 Lee I. This article is distributed under the terms of the Creative Commons Attribution 4.0 International License.

\section{Keywords:}

Aortic dissection

STEMI

Stroke

Cardiogenic shock

Spinal cord syndrome

\section{Abstract}

Background: Identifying and treating ST-elevation myocardial infarction (STEMI) in a timely manner is crucial in reducing the patient's morbidity and mortality. However, sometimes STEMI can be caused by other pathologies such as aortic/coronary dissection, thromboembolism, septic emboli, or trauma. We present a case of a pan-aortic dissection (AD) with multi-system organ failure and shock presenting as anterior spinal cord syndrome, inferior myocardial infarction and stroke.

Case Presentation: A 62-year old woman with history of tobacco abuse and depression, presented to the Emergency Department (ED) for altered mental status. The patient was initially found outside of her home, unresponsive and bradycardic, with subsequent 12-lead electrocardiogram (EKG) demonstrating an inferior STEMI with right ventricle (RV) involvement and presumed cardiogenic shock. In the ED, the patient was awake with intermittent, though profound aphasia, as well as near complete loss of motor and sensory function in her lower extremities. She remained hypotensive and had weak peripheral pulses bilaterally in the upper and lower extremities. Emergent brain imaging was negative for ischemia, but chest imaging revealed circumferential mural thrombus extending from the aortic root into the descending aorta consistent with pan- $A D$ and was taken urgently to the operating suite. Unfortunately, the patient died before surgical intervention could be performed.

Conclusion: This case report highlights a rare case of STEMI, stroke, cardiogenic shock and ultimately death caused by pan-AD. Timely identifying the underlying pathology in patients who present with more than typical STEMI symptoms is essential in providing potential lifesaving therapy.

\section{Learning Points}

- Aortic dissection symptoms can present with cardiovascular \& neurological symptoms, end-organ compromise along with chest and/or abdominal pain.

- Clinicians should have high degrees of suspicion when myriad of symptoms are present in hemodynamically unstable patients.

- Aortic dissection can have numerous complications including death if not properly diagnosed and treated accordingly.

- Treatments of aortic dissection differs based on its type, hemodynamic status of the patient and any signs of end organ dysfunction.

\section{Background}

Thoracic and abdominal AD can present in a myriad of ways, 
with symptoms reflecting locations of end-organ damage. Most individuals present with a similar triad of hypertension, tachycardia and pain. However, given the relative infrequency of presentation (5-30 cases $/ 1,000,000$ patients), individuals more often have vague, non-specific symptoms, making the diagnosis challenging ${ }^{1,2}$. It has been reported that nearly $40 \%$ of $\mathrm{AD}$ are missed at the time of presentation and $30 \%$ discovered postmortem ${ }^{1,2}$, with delays in care owing to a high mortality risk ${ }^{3-5}$. For this reason, physicians must have a high clinical index of suspicion, especially in the setting of progressive combined cardiovascular and neurologic symptoms that are unexpected in the evolution of a broad differential diagnosis.

\section{Case Presentation}

A 62-year old female with a past medical history of tobacco abuse and depression, presented to the ED for altered level of consciousness and initial reports from emergency medical service (EMS) were concerning for a stroke. Earlier that day, the patient called EMS with a chief complaint of bilateral lower extremity numbness, tingling and weakness but upon arrival to the patient's home over twenty minutes later, the patient was found outside, minimally responsive with bilateral lower extremity paraplegia. Vital signs in the field were significant for hypotension and bradycardia. A 12-lead EKG was obtained in the field and the presumptive diagnosis of cardiogenic shock secondary to an inferior STEMI with RV involvement (Figure A) was made. The patient was given $324 \mathrm{mg}$ of acetylsalicylic acid and $0.5 \mathrm{mg}$ of atropine on her way to the hospital.

Initial vital signs in the ED demonstrated a heart rate of $58 \mathrm{bpm}$ and a blood pressure of $62 / 40 \mathrm{mmHg}$. Her physical examination was pertinent for bounding carotid pulse, diminished breath sounds bilaterally, and cool extremities with severely diminished lower extremity peripheral pulses. Neurologically, the patient maintained the ability to open her eyes and maintained normal upper extremity movement and grip strength but could only speak intermittently, denying pain. Additionally, the patient's lower extremities were noted to be pale and did not withdraw to pain.

Basic labs were obtained prior to imaging and were significant for a shite blood cell count of $11.8 \mathrm{~K} /{ }^{\mu} \mathrm{L}(\mathrm{Ref}$ 4.5 to $11.0 \mathrm{~K} /{ }^{\mu} \mathrm{L}$ ), hematocrit of $37.4 \%$ (Pof 37-48\%), platelet count of $111 \mathrm{~K} / \mu_{\mathrm{L}}\left(\operatorname{Ref} 150-450 \mathrm{~K} / \mu_{\mathrm{L}}\right)$, a serum creatinine $1.00 \mathrm{mg} / \mathrm{dL}$ (Ref 0.5-1.1 mg/dL) which was elevated from her previous baseline of $0.6 \mathrm{mg} / \mathrm{dL}$, troponin I negative, and an international normalized ratio (INR) of $2.0($ Ref $<1.1)$ without outpatient anticoagulant use.

Initial standard acute coronary syndrome (ACS) measures were withheld given her atypical presentation and emergent imaging was obtained. Cerebral imaging was negative for ischemia, but computerized tomography (CT) scans of the chest and abdomen revealed circumferential mural thrombus extending from the aortic root and arch with minimal flow through the brachiocephalic branch vessels and distal extension into the descending abdominal aorta (Figures B 1-5). Cardiothoracic surgery was consulted for urgent intervention, but the patient suffered cardiac arrest upon entering the operating room and an intra-code transesophageal echocardiogram (TEE) after intubation was negative for intrinsic cardiac contractility, at which time the patient was pronounced dead.

\section{Discussion and Conclusions}

The clinical presentation of acute thoracic or abdominal aortic dissection is one that can prove to be a diagnostic challenge when dealing with perplexing, multi-organ system physical examination findings. However, even a good clinical history may not yield to an underlying diagnosis because of diverse nature of intitial presenting symptoms

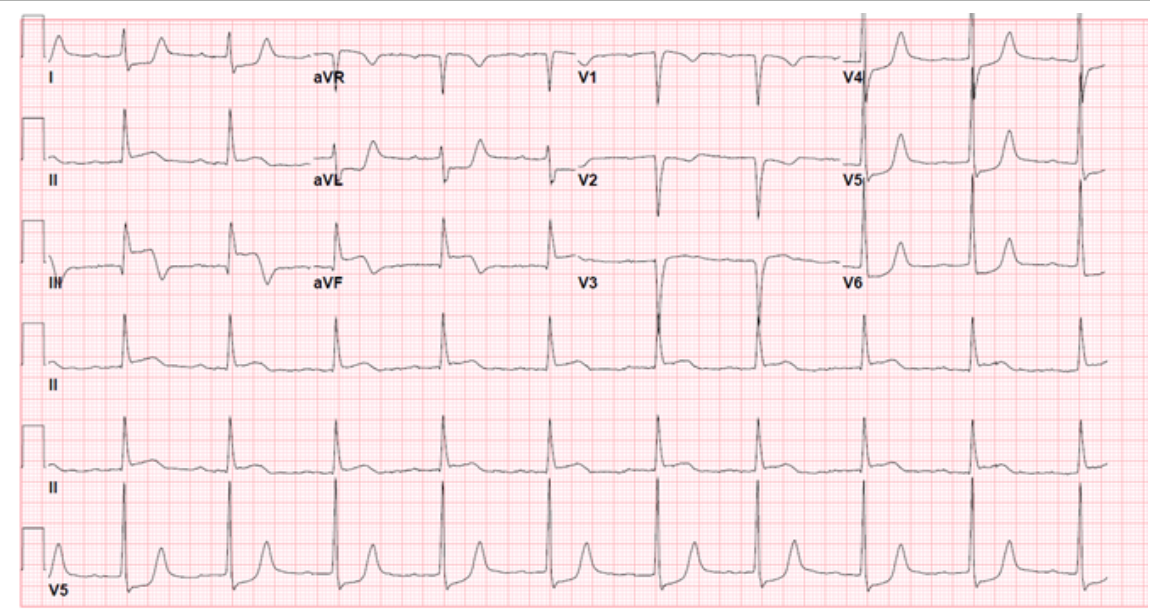

Figure A. Initial EKG in the field consistent with inferior myocardial infarction with right ventricle involvement, likely involving the proximal right coronary artery (RCA). 


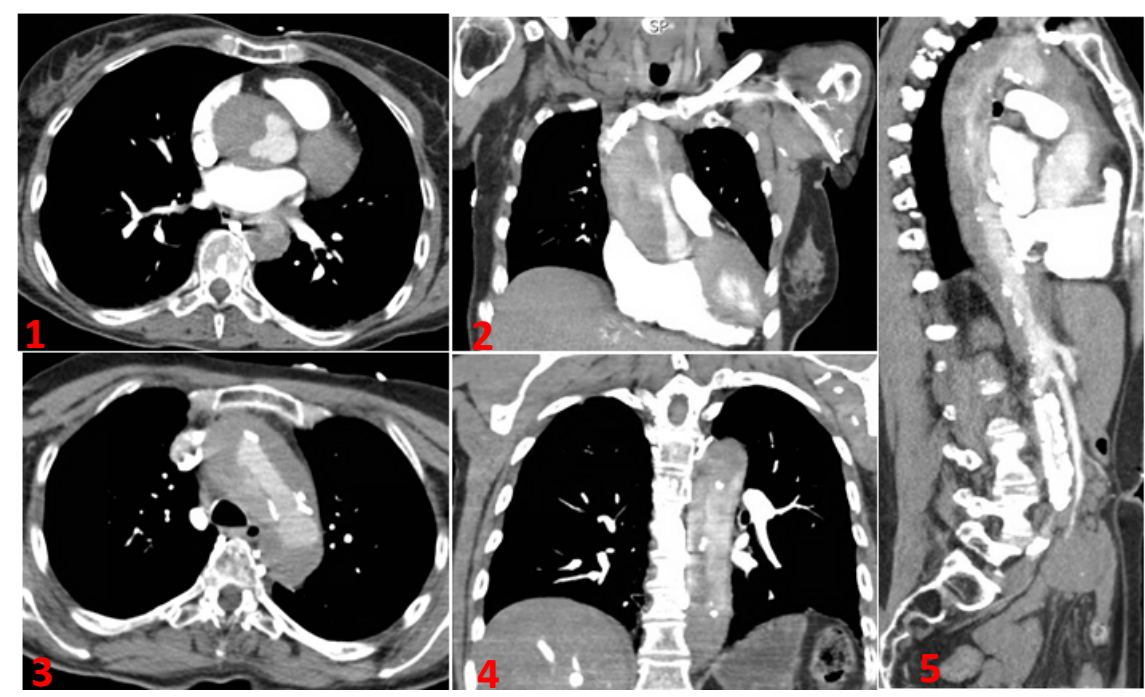

Figure B 1-5. Contrast CT imaging of the chest demonstrating the proximal nature of dissection with involvement of the aortic root $(1,2)$, arch (3), descending thoracic $(4,5)$, and descending thoracic and abdominal aorta (5).

that can bias early decision making. In this situation, the combination of initial peripheral neurologic symptoms suggestive of spinal cord involvement, EKG changes consistent with ACS, and ultimately cerebrovascular impairment secondary to hypoperfusion, represented a triad of catastrophic end-organ mainfestations in the same patient.

The most common historical finding with $\mathrm{AD}$ is pain, typically in the chest, abdomen or back ${ }^{4}$. It has been reported that up to $95 \%$ of all patients with dissection report pain, and $85 \%$ of those individuals described it as abrupt in onset $^{4,6}$. This pain is described as "tearing" or "ripping," but is described as such in only $50 \%$ of the individuals ${ }^{5,7}$. However, up to $10 \%$ of patient's with dissection present without pain, complicating the overall picture and may be misleading and delay definitive diagnosis ${ }^{2}$. Without typical symptoms and a high index of suspician, clinicians are left with only vague symptoms and a broad differential. In our patient, pain was not a symptom elicited in part due to the cerebrovascular complications and aphasia on presentation, as well as the disjointed timecourse with nearly an hour before she was evaluated by the EMS.

Hemodynamics are a key feature for most clinicians when evaluating for the possibility of $\mathrm{AD}$, but are often commonly misinterpreted. While we typically think of dissection presenting with hypertension, up to one fourth of individuals, especially those with proximal involvement, present with hypotension of multi-factorial etiology ${ }^{6,7}$. These patients typically have the worst prognosis and are associated with catastrophic involvement of the heart by way of aortic valve pathology, left ventricular failure or tamponade physiology $y^{4,6,8,9,10}$. Another common hemodynamic parameter that can heighten clinical suspicion is the discrepancies in pulses and blood pressure of the upper and lower extremities, which is the most specific physical finding in proximal $\mathrm{AD}$ involving the subclavian artery and has been found in up to $38 \%$ of the patients $^{4,8}$.

Although, plan radiography can suggest subtle signs of $\mathrm{AD}$, advanced imaging techniques such as CT angiography (CTA) or MRI, have much higher sensitivity for detecting dissection than plain films ${ }^{11-13}$. CTA has been recommended as the imaging technique of choice by the American College of Radiology because in high and intermediate risk patients as defined in previous sections, CTA demonstrates sensitivity and specificity of $83-94 \%$ and $87-100 \%$, respectively ${ }^{13,14}$. MRI has been shown to be marginally better than CTA, demonstrating a 98 - 100\% specificity and sensitivity for AD in multiple clinical trials ${ }^{15,16}$. Besides a high sen/spec, imaging with MRI does not require contrast and is ideal for patients with renal failure or those with allergies to contrast dye. However, because of the longer scanning time and lack of availability at some medical centers, this is typically not recommended in the triage evaluation of patients with intermediate or high probability. In the past, it has been said that these imaging techniques, which clearly define anatomic features with stunning accuracy, they cannot help in determining the etiology of hemodynamic instability that can help drive clinical decision making.

The same discussion can be held for TEE, which is routinely employed if proximal dissection is suspected. One of the biggest advantages of TEE over other imaging techniques, is that it is portable and can be performed at the bedside, making it ideal for hemodynamically unstable patients ${ }^{17,18}$. Recent literature has demonstrated a sensitivity and specificity of $97-99 \%$ and $97-100 \%$, respectively, for TEE determination of aortic root dissection 
and related complications ${ }^{17}$. Contrast this modality with the similar transthoracic echocardiography (TTE), with wide ranging sensitivities and specificities of $35-80 \%$ and $36-96 \%$, respectively, and is by no means a sufficient test to rule out the presence of $\mathrm{AD}^{4}$. In our case, due to her rapid clinical deterioration prior to echocardiographic evaluation, a thorough TEE could not be accomplished.

End organ involvement can be present in up to $30 \%$ of individuals, with heart being the most common bystander ${ }^{8,9}$. Evidence of myocardial ischemia, whether from coronary supply-demand mismatch, direct extension of the dissection, extrinsic vessel compression or extravasation leading to mass effect, is a common finding ${ }^{7,8}$. In our case, patient presented with an inferior STEMI involving the right coronary artery (RCA) with initial intentions for emergent cardiac catheterization or treatment with thrombolysis and antiplatelet agents, which could have hastened her decompensation.

Neurologic involvement is a prominent feature representing upwards of $20-30 \%$ of dissection cases and are delineated into cerebrovascular, spinal or autonomic etiologies $^{2,5}$. For some individuals, this may represent a meretransient drop in perfusion to the affected regions and up to $12 \%$ of the patients may present with a single syncopal event ${ }^{2,4,5}$. Proximal aortic involvement may result in cerebral hypoperfusion secondary to the same mechanisms as myocardial ischemia described above. Typically, distal aortic involvement may present as an anterior spinal syndrome secondary to the involvement of watershed area between the territories of the artery of Adamkiewicz and the thoracic radicular artery, which we believe was the etiology of our patient's paraplegia and initial radicular symptoms ${ }^{2,4}$. Autonomic or peripheral nerve involvement is the least encountered clinical finding and is typically overlooked in the more severe presentations of AD. When neurological symptoms are present, the most commonly described symptoms are stroke, syncope or hoarseness of the voice from recurrent laryngeal nerve compression around the aortic arch ${ }^{2}$.

AD represents a potentially deadly condition with high degree of variability in symptoms that can be challenging for physicians to diagnose. Thus, physicians need to be aware of the relatively subtle ways in which $\mathrm{AD}$ can present. Our patient presented as a STEMI alert with unusual clinical findings that were secondary to panAD with clinical and radiographic evidence of end organ involvement and atypical hemodynamics. STEMI is usually a primary diagnosis for most patients, however, our patient presented with pan-aortic dissection that resulted in STEMI, cardiogenic shock and stroke symptoms. When STEMI is caused by a different pathology than the well-known plaque rupture presentation, a secondary cause should be investigated and treated promptly ${ }^{1}$. Given the significant morbidity and mortality associated with delayed treatment of STEMI, it is easy to overlook the symptoms that may hint towards a different pathology. This case report highlights the importance of having high clinical suspicion when STEMI patients are presented with variety of symptoms other than the typical ACS signs.

\section{Declarations}

Ethics approval and consent to participate: The manuscript strictly adheres to the ethics committee guidelines.

Consent for Publication: No next of kin was available and the patient was deceased. Consent was obtained from the hospital and the doctor to use the patient's medical record.

Availability of data and material: All datasets used for supporting this article are available from the Veterans Affairs Medical Center database.

\section{Competing interests: None declared}

Funding: There was no specific grant for this research from any funding agency in the public, commercial or notfor-profit sectors.

Authors' contributions: All authors contributed in this case report. IL is the cardiology fellow who managed the research protocol and wrote the report. MC is the cardiology fellow that oversaw the patient and provided the figures as well as any pertinent data. AP is the attending cardiologist who directly took care of this patient.

Acknowledgements: For our patient, who passed away with pan-aortic dissection.

\section{References}

1. Lentini S, Perrotta S. Aortic dissection with concomitant acute myocardial infarction: From diagnosis to management. J Emerg Trauma Shock. 2011; 4: 273-8.

2. Spittell PC, 2JA Jr, Joyce JW, Tajik AJ, et al. Clinical features and differential diagnosis of aortic dissection Experience with 236 cases 1980 through 1990. May Clin Proc. 1993; 68: 642-51.

3. Isselbacher EM. Contemporary reviews in cardiovascular medicine: Thoracic and abdominal aortic aneurysms. Circulation. 2005; 111: 816-828.

4. Khan IA, Nair CK. Clinical, diagnostic, and management perspectives of aortic dissection. CHEST. 2002; 122: 311-328.

5. Von Kodolitsch Y, Schwartz AG, Nienaber CA. Clinical Prediction of acute aortic dissection. Arch Intern Med. 2000; 160: 2977.

6. Hagan PG, Nienaber CA, 3EM, et al. The International Registry of Acute Aortic Dissection (IRAD): new insights into an old disease. JAMA. 2000; 283: 897-903.

7. Meszaros I, Morocz J, Szlavi J, et al. Epidemiology and clinicopathology of aortic dissection. Chest. 2000; 117: 1271-1278.

8. Braverman AC. Clinician update Acute aortic dissection. Circulation. 2010; 122: 184-188.

9. Garcia-Jimenez A, Peraza-Torres A, Martinez-Lopez G, et al. Cardiac 
tamponade by aortic dissection in a hospital without cardiothoracic surgery. Chest. 1993; 104: 290-291.

10. Patel YD. Rupture of an aortic dissection into the pericardium. Cardiovasc Intervent Radiol. 1986; 9: 222-224.

11. Earnest F, Muhm JR, Sheedy PF. Roentgenographic findings in thoracic aortic dissection. Mayo Clin Proc. 1979; 54: 43-50.

12. Jagannath AS, Sos TA, Lockhart SH, et al. Aortic dissection: a statistical analysis of the usefulness of plain chest radiographic findings. Am J Roentgenol. 1986; 147: 1123-1126.

13. Mammen L, Yucel EK, 4A, et al. American College of Radiology Appropriateness Criteria acute chest pain - suspected aortic dissection. 2008; 2-7.
14. Treasure T, Raphael MJ. Investigation of suspected dissection of the thoracic aorta. Lancet. 1991; 338: 490-494.

15. Nienaber CA, $5 Y$, Nicolas $\mathrm{V}$, et al. The diagnosis of thoracic aortic dissection by noninvasive imaging procedures. $\mathbf{N}$ Engl J Med. Jan 7 1993; 328(1): 1-9.

16. Nienaber CA, Spielmann RP, 5Y, et al. Diagnosis of thoracic aortic dissection. Magnetic resonance imaging versus transesophageal echocardiography. Circulation. Feb 1992; 85(2): 434-47.

17. Meredith EL, Masani ND. Echocardiography in the emergency assessment of acute aortic syndromes. Eur J Echocardiogr. Jan 2009; 10(1): i31-9.

18. Erbel R, Engberding R, Daniel W, et al. Echocardiography in diagnosis of aortic dissection. Lancet. Mar 4 1989; 1(8636): 457-61. 